\title{
A class of marked invariant subspaces with an application to algebraic Riccati equations
}

\author{
Pudji Astuti \\ Harald K. Wimmer \\ Faculty of Mathematics and \\ Mathematisches Institut \\ Natural Sciences \\ Universität Würzburg \\ Institut Teknologi Bandung \\ Bandung 40132 \\ D-97074 Würzburg \\ Germany \\ Indonesia
}




\begin{abstract}
Mathematical Subject Classifications (2010): 15A18, 47A15 15A24, $15 \mathrm{~B} 57$.

Keywords: invariant subspaces, Jordan chains, marked subspaces, algebraic Riccati equation, Hamiltonian matrix.

Abstract: Invariant subspaces of a matrix $A$ are considered which are obtained by truncation of a Jordan basis of a generalized eigenspace of $A$. We characterize those subspaces which are independent of the choice of the Jordan basis. An application to Hamilton matrices and algebraic Riccati equations is given.
\end{abstract}




\section{Invariant subspaces}

Let $\lambda$ be an eigenvalue of a complex $n \times n$ matrix $A$ and let

$$
E_{\lambda}(A)=\operatorname{Ker}(A-\lambda I)^{n}
$$

be the corresponding generalized eigenspace. Suppose $\operatorname{dim} E_{\lambda}(A)=k$. If

$$
(s-\lambda)^{t_{1}}, \ldots,(s-\lambda)^{t_{k}}, t_{1} \leq \cdots \leq t_{k},
$$

are the corresponding elementary divisors then $E_{\lambda}(A)$ is a direct sum of $t_{i}$-dimensional cyclic subspaces, i.e.

$$
E_{\lambda}(A)=K_{1} \oplus \cdots \oplus K_{k}
$$

with

$$
K_{i}=\operatorname{span}\left\{u_{i},(A-\lambda I) u_{i}, \ldots,(A-\lambda I)^{t_{i}-1} u_{i}\right\},
$$

and $(A-\lambda I)^{t_{i}} u_{i}=0, \quad i=1, \ldots, k$. We call

$$
U=\left(u_{1}, \ldots, u_{k}\right)
$$

a tuple of generators of $E_{\lambda}(A)$. From a given $U$ one can construct $A$-invariant subspaces in the following way. Let $r=\left(r_{1}, \ldots, r_{k}\right)$ be such that

$$
0 \leq r_{i}<t_{i}, i=1, \ldots, k \text {. }
$$

We set

$$
W_{r_{i}}(U)=\operatorname{span}\left\{(A-\lambda I)^{r_{i}} u_{i},(A-\lambda I)^{r_{i}+1} u_{i}, \ldots,(A-\lambda I)^{t_{i}-1} u_{i}\right\}
$$

and

$$
W(r, U)=W_{r_{1}}(U) \oplus \cdots \oplus W_{r_{k}}(U) .
$$

The construction of invariant subspaces of the from $W(r, U)$ is a standard procedure in linear algebra and systems theory (see e.g. [8], [6, p.61], [5], $[10],[2$, p.28]).

If $U$ and $\tilde{U}$ are two different tuples of generators of $E_{\lambda}(A)$ then the restrictions of $A$ to $W(r, U)$ and $W(r, \tilde{U})$ have the same elementary divisors, namely $(s-\lambda)^{t_{i}-r_{i}}, i=1, \ldots, k$. However, in general, the subspaces $W(r, U)$ and $W(r, \tilde{U})$ will be different. Consider the following example with $k=2$, $t_{1}=2, t_{2}=3$, and

$$
A=\operatorname{diag}\left(N_{2}, N_{3}\right), N_{2}=\left(\begin{array}{ll}
0 & 1 \\
0 & 0
\end{array}\right), N_{3}=\left(\begin{array}{lll}
0 & 1 & 0 \\
0 & 0 & 1 \\
0 & 0 & 0
\end{array}\right) .
$$


Let $e_{i}$ be a unit vector of $\mathbb{C}^{5}$. Then $U=\left\{e_{2}, e_{5}\right\}$ and $\tilde{U}=\left\{e_{2}, e_{5}+e_{2}\right\}$ are tuples of generators of $E_{0}(A)=\operatorname{Ker} A^{5}=\mathbb{C}^{5}$. If we choose $r=(1,0)$, then $W(r, U)=\operatorname{span}\left\{e_{1}, e_{3}, e_{4}, e_{5}\right\}$ and $W(r, \tilde{U})=\operatorname{span}\left\{e_{1}, e_{3}, e_{4}+e_{1}, e_{5}+e_{2}\right\}$. Thus

$$
W(r, U) \neq W(r, \tilde{U}) .
$$

On the other hand, if we choose $r=(1,2)$, then

$$
W(r, U)=W(r, \tilde{U}) .
$$

It is the purpose of our note to determine those tuples $r=\left(r_{1}, \ldots, r_{k}\right)$ which have the property that the space $W(r, U)$ given by (1.4) and (1.5) is independent of the generator tuple $U$. The motivation for our study comes from Kucera's survey article [8], which deals with independence of generator tuples in the case of Hamiltonian matrices. In Section 3 we make the connection with $[8$, p.60] applying a corollary of our main theorem to Hamiltonian matrices and algebraic Riccati equations.

In the sequel we assume that $\lambda=0$ is an eigenvalue of $A$ and we focus on $E_{0}(A)=\operatorname{Ker} A^{n}$. With each nonzero vector $v \in E_{0}(A)$ we associate a height $\mathrm{h}(v)$ and an exponent $\mathrm{e}(v)$ as follows. Suppose

$$
v \in \operatorname{Im} A^{q}, v \notin \operatorname{Im} A^{q+1}, v \in \operatorname{Ker} A^{p}, v \notin \operatorname{Ker} A^{p-1} .
$$

Then we set $\mathrm{h}(v)=q$ and $\mathrm{e}(v)=p$. Thus, if $\lambda=0$ in (1.1) then the elements of $U$ in (1.2) satisfy $\mathrm{e}\left(u_{1}\right)=t_{1} \leq \cdots \leq \mathrm{e}\left(u_{k}\right)=t_{k}$ and $\mathrm{h}\left(u_{i}\right)=0$. We define

$$
\langle v\rangle=\operatorname{span}\left\{A^{\nu} v, \nu \geq 0\right\} .
$$

Then $\langle v\rangle$ is a cyclic subspace generated by $v$, and $\operatorname{dim}\langle v\rangle=\mathrm{e}(v)$.

\section{The main result}

Theorem 2.1. Let $A \in \mathbb{C}^{n \times n}$ and let

$$
s^{t_{1}}, \ldots, s^{t_{k}}, t_{1} \leq \cdots \leq t_{k}
$$

be the elementary divisors corresponding to the eigenvalue $\lambda=0$. Let

$$
U=\left(u_{1}, \ldots, u_{k}\right)
$$

be a tuple of generators of $E_{0}(A)=\operatorname{Ker} A^{n}$ such that $\mathrm{e}\left(u_{i}\right)=t_{i}, i=1, \ldots, k$, and

$$
E_{0}(A)=\left\langle u_{1}\right\rangle \oplus \cdots \oplus\left\langle u_{k}\right\rangle
$$


Let $r=\left(r_{1}, \ldots, r_{k}\right)$ be a $k$-tuple of integers with $0 \leq r_{i}<t_{i}, i=1, \ldots, k$. Define

$$
W(r, U)=\left\langle A^{r_{1}} u_{1}\right\rangle \oplus \cdots \oplus\left\langle A^{r_{k}} u_{k}\right\rangle
$$

and

$$
W(r)=\left(\operatorname{Im} A^{r_{1}} \cap \operatorname{Ker} A^{t_{1}-r_{1}}\right)+\cdots+\left(\operatorname{Im} A^{r_{k}} \cap \operatorname{Ker} A^{t_{k}-r_{k}}\right) .
$$

Then the following statements are equivalent:

(i) The $k$-tuple $r=\left(r_{1}, \cdots, r_{k}\right)$ satisfies

$$
r_{1} \leq \cdots \leq r_{k}
$$

and

$$
t_{1}-r_{1} \leq \cdots \leq t_{k}-r_{k} .
$$

(ii) The space $W(r, U)$ is independent of $U$.

Moreover, if (2.4) and (2.5) hold then $W(r, U)=W(r)$.

Proof. (i) $\Rightarrow$ (ii). We show that (2.4) and (2.5) imply $W(r, U)=W(r)$. Define $W_{r_{s}}(U)=\left\langle A^{r_{s}} u_{s}\right\rangle$ such that (1.5) holds. From

$$
W_{r_{s}}(U) \subseteq \operatorname{Im} A^{r_{s}} \cap \operatorname{Ker} A^{t_{s}-r_{s}}
$$

we immediately obtain $W(r, U) \subseteq W(r)$. Now let $x$ be in $\operatorname{Im} A^{r_{s}} \cap \operatorname{Ker} A^{t_{s}-r_{s}}$. Then $x=A^{r_{s}} y$ for some $y \in E_{0}(A)$, and

$$
A^{t_{s}-r_{s}} x=A^{t_{s}} y=0 .
$$

With respect to the basis

$$
\mathcal{B}_{U}=\left\{A^{\nu_{i}} u_{i} ; 0 \leq \nu_{i} \leq t_{i}-1, i=i, \ldots, k\right\}
$$

we have

$$
y=\sum_{i=1}^{k} \sum_{\nu_{i}=0}^{t_{i}-1} \alpha_{i \nu_{i}} A^{\nu_{i}} u_{i} .
$$

Let $\ell$ be the largest integer sucht that $t_{\ell} \leq t_{s}$. Then $A^{t_{s}} u_{i}=0$ for $i=$ $1, \ldots, \ell$. Moreover $A^{t_{s}+\nu_{i}} u_{i}=0$ if $t_{s}+\nu_{i}>t_{i}$. Therefore

$$
A^{t_{s}} y=\sum_{i>\ell} \sum_{\nu_{i}=0}^{t_{i}-t_{s}-1} \alpha_{i \nu_{i}} A^{t_{s}+\nu_{i}} u_{i}=0
$$


Since the vectors of $\mathcal{B}_{U}$ are linearly independent we obtain $\alpha_{i \nu_{i}}=0$ for $i>\ell$ and $\nu_{i}=0, \ldots, t_{i}-t_{s}-1$. Hence

$$
y=\sum_{i=1}^{\ell} \sum_{\nu_{i}=0}^{t_{i}-1} \alpha_{i \nu_{i}} A^{\nu_{i}} u_{i}+\sum_{i>\ell} \sum_{\nu_{i}=t_{i}-t_{s}}^{t_{i}-1} \alpha_{i \nu_{i}} A^{\nu_{i}} u_{i}
$$

and

$$
x=\sum_{i=1}^{\ell} \sum_{\nu_{i}=0}^{t_{i}-1} \alpha_{i \nu_{i}} A^{r_{s}+\nu_{i}} u_{i}+\sum_{i>\ell} \sum_{\nu_{i}=t_{i}-t_{s}}^{t_{i}-1} \alpha_{i \nu_{i}} A^{r_{s}+\nu_{i}} u_{i} .
$$

Note that $t_{s}=\cdots=t_{\ell}$ implies $r_{s}=\cdots=r_{\ell}$. Hence, if $1 \leq i \leq \ell$ then $r_{i} \leq r_{s}$, and therefore

$$
A^{r_{s}+\nu_{i}} u_{i} \in W_{r_{i}}(U) .
$$

On the other hand, if $i>\ell$ then $t_{s}-r_{s} \leq t_{i}-r_{i}$. In that case $\nu_{i} \in$ $\left\{t_{i}-t_{s}, \ldots, t_{i}-1\right\}$ implies

$$
r_{s}+\nu_{i} \geq r_{s}+\left(t_{i}-t_{s}\right) \geq r_{i}
$$

Thus, we again have (2.8). Hence $x \in W(r, U)$ and therefore $W(r) \subseteq$ $W(r, U)$.

(ii) $\Rightarrow$ (i). We assume that $W(r, U)$ is independent of $U$. Let us show first that

$$
r_{i}=r_{j} \text { if } t_{i}=t_{j}
$$

Suppose $r=\left(r_{1}, \ldots, r_{k}\right)$ is such that $t_{s}=t_{s+1}$ and $r_{s} \neq r_{s+1}$, e.g.

$$
r_{s+1}<r_{s} \text { for some } s \in\{1, \ldots, k-1\} \text {. }
$$

Let $V=\left(v_{1}, \ldots, v_{k}\right)$ be such that $\left(v_{s}, v_{s+1}\right)=\left(u_{s+1}, u_{s}\right)$, and $v_{i}=u_{i}$ if $i \notin\{s, s+1\}$. Then $A^{r_{s+1}} u_{s+1} \in W(r, U)$ but $A^{r_{s+1}} u_{s+1}=A^{r_{s+1}} v_{s} \notin W(r, V)$. Therefore the tuples $U$ and $V$ contain the same elements, but $W(r, U) \neq$ $W(r, V)$.

Now suppose that (2.4) is not satisfied. Then we have (2.10), and

$$
A^{r_{s+1}} u_{s} \notin W(r, U) .
$$

Let $V=\left(v_{1}, \ldots, v_{k}\right)$ be given by $v_{s+1}=u_{s+1}+u_{s}$, and $v_{i}=u_{i}$, if $i \neq s+1$. Thus $V$ is a tuple of generators of $E_{0}(A)$ with $\mathrm{e}\left(v_{i}\right)=\mathrm{e}\left(u_{i}\right)$. Consider

$$
A^{r_{s+1}} u_{s+1}+A^{r_{s+1}} u_{s}=A^{r_{s+1}} v_{s+1} \in W(r, V) .
$$

Then $A^{r_{s+1}} v_{s+1} \notin W(r, U)$. Otherwise $A^{r_{s+1}} u_{s+1} \in W(r, U)$ would imply $A^{r_{s+1}} u_{s} \in W(r, U)$, which is a contradiction to (2.11). 
Suppose $r=\left(r_{1}, \ldots, r_{k}\right)$ does not satisfy (2.5). Then $t_{s}-r_{s}>t_{s+1}-r_{s+1}$ for some $s \in\{1, \ldots, k-1\}$. Because of $(2.9)$ we have $t_{s+1} \neq t_{s}$. Hence $r_{s+1}-r_{s}>t_{s+1}-t_{s}>0$, and $r_{s}<r_{s+1}$, and

$$
r_{s}+\left(t_{s+1}-t_{s}\right)<r_{s+1}
$$

Because (2.2) it is obvious that (2.12) implies

$$
A^{r_{s}+\left(t_{s+1}-t_{s}\right)} u_{s+1} \notin W(r, U) .
$$

Define $v_{s}=u_{s}+A^{t_{s+1}-t_{s}} u_{s+1}$. Then $\mathrm{e}\left(v_{s}\right)=\mathrm{e}\left(u_{s}\right)=t_{s}$. Therefore

$$
V=\left\{u_{1}, \ldots, u_{s-1}, v_{s}, u_{s+1}, \ldots, u_{k}\right\}
$$

is another tuple of generators of $E_{0}(A)$. Let us show that $W(r, V) \neq W(r, U)$. Clearly, the vector $A^{r_{s}} v_{s}$ belongs to $W(r, V)$. Suppose

$$
A^{r_{s}} u_{s}+A^{r_{s}+\left(t_{s+1}-t_{s}\right)} u_{s+1}=A^{r_{s}} v_{s} \in W(r, U) .
$$

Because of $A^{r_{s}} u_{s} \in W(r, U)$ that would imply

$$
A^{r_{s}+\left(t_{s+1}-t_{s}\right)} u_{s+1} \in W(r, U),
$$

which is a contradiction to (2.13).

Let us consider again Example (1.6). We have $\left(t_{1}, t_{2}\right)=(2,3)$. In the case of $r=(1,0)$ condition (2.4) is violated, which accounts for (1.7). In the case of $r=(1,2)$ both (2.4) and (2.5) hold, which ensures (1.8).

In accordance with a definition in $[7$, p. 83] and [3] the space $W(r, U)$ is a marked $A$-invariant subspace of $E_{0}(A)$. That means $W(r, U)$ has a Jordan basis, in our case

$$
\left\{A^{r_{i}+\mu_{i}} u_{i} ; 0 \leq \mu_{i} \leq t_{i}-r_{i}-1, i=i, \ldots, k\right\}
$$

which can be extended to a Jordan basis of $E_{0}(A)$, namely to $\mathcal{B}_{U}$ in (2.7). Let $\mathcal{M}_{r}$ be the set of marked subspaces $M$ of $E_{0}(A)$ such that the elementary divisors of the restriction $A_{\mid M}$ are $s^{t_{1}-r_{1}}, \ldots, s^{t_{k}-r_{k}}$. We have noted before that for each tuple of generators $U$ the corresponding space $W(r, U)$ is in $\mathcal{M}_{r}$. Suppose (2.4) and (2.5) hold. Then all the spaces $W(r, U)$ coincide with $W(r)$ and one might ask whether $W(r)$ is the only subspaces in $\mathcal{M}_{r}$. In the following we have an example where $\mathcal{M}_{r} \supsetneqq\{W(r)\}$. Let $n=10$, $k=2$, and $t=\left(t_{1}, t_{2}\right)=(4,6)$, and $r=(2,3)$. Then $t-r=(2,3)$. Hence 
the conditions (2.4) and (2.5) are satisfied. Let $U=\left(u_{1}, u_{2}\right)$ be a tuple of generators such that $\mathrm{e}\left(u_{1}\right)=4$ and $\mathrm{e}\left(u_{2}\right)=6$. The subspaces

$$
M=W(r, U)=W(r)=\left\langle A^{2} u_{1}\right\rangle \oplus\left\langle A^{3} u_{2}\right\rangle
$$

and $\tilde{M}=\left\langle A u_{1}\right\rangle \oplus\left\langle A^{4} u_{2}\right\rangle$ are marked, the elementary divisors of $A_{\mid M}$ and $A_{\mid \tilde{M}}$ are $s^{2}, s^{3}$. Hence $\tilde{M} \in \mathcal{M}_{r}$, but $\tilde{M} \neq W(r)$.

Let $[m]$ denote the greatest integer of $m$. If we assume $\left(t_{1}, \ldots, t_{k}\right)$ as in (2.1) and take $r=\left(\left[\frac{1}{2} t_{1}\right], \ldots,\left[\frac{1}{2} t_{k}\right]\right)$ then the conditions (2.4) and (2.5) are satisfied and we note the following corollary of Theorem 2.1.

Corollary 2.2. Let $A \in \mathbb{C}^{n \times n}$ and $0 \in \sigma(A)$. Let $s^{2 m_{1}}, \ldots, s^{2 m_{k}}$, be the elementary divisors of $A$ corresponding to $\lambda=0$. If $U=\left(u_{1}, \ldots, u_{k}\right)$ is a tuple of generators of $\operatorname{Ker} A^{n}$ such that $\mathrm{e}\left(u_{i}\right)=2 m_{i}, i=1, \ldots, k$, then $\mathrm{e}\left(A^{m_{i}} u_{i}\right)=m_{i}$ for all $i$, and

$$
\left\langle A^{m_{1}} u_{1}\right\rangle \oplus \cdots \oplus\left\langle A^{m_{k}} u_{k}\right\rangle=\left(\operatorname{Im} A^{m_{1}} \cap \operatorname{Ker} A^{m_{1}}\right)+\cdots+\left(\operatorname{Im} A^{m_{k}} \cap \operatorname{Ker} A^{m_{k}}\right) .
$$

\section{An application}

In this section we apply Corollary 2.2 to the algebraic Riccati equation

$$
Q+F^{*} X+X F-X D X=0
$$

and its associated Hamiltonian matrix

$$
H=\left(\begin{array}{cc}
F & -D \\
-Q & -F^{*}
\end{array}\right)
$$

Here $F, D, Q$ are complex $m \times m$ matrices, $D$ and $Q$ are hermitian, $D \geq 0$, and the pair $(F, D)$ is assumed to be controllable. Then (see [8, p.59] all elementary divisors corresponding to eigenvalues $i \alpha \in i \mathbb{R}$ have even degree. To fix ideas we assume $\sigma(H)=\{0\}$. The subsequent result complements Lemma 3.2.3 of [8, p.60].

Proposition 3.1. Let $s^{2 m_{1}}, \ldots s^{2 m_{k}}$ be the elementary divisors of $H$. Set

$$
W=\left(\operatorname{Im} H^{m_{1}} \cap \operatorname{Ker} H^{m_{1}}\right)+\cdots+\left(\operatorname{Im} H^{m_{k}} \cap \operatorname{Ker} H^{m_{k}}\right) .
$$

Then $W$ is an $H$-invariant subspace of $\mathbb{C}^{2 m}$ and $\operatorname{dim} W=m$. Let $Y, Z \in$ $\mathbb{C}^{m \times m}$ be such that the columns of $(\underset{Z}{Y})$ are a basis of $W$. Then $Y$ is nonsingular and $X=Z Y^{-1}$ is the unique hermitian solution of (3.1). 
Proof. Set $t=\left(2 m_{1}, \ldots, 2 m_{k}\right)$. Let $U=\left(u_{1}, \ldots, u_{k}\right)$ be a tuple of generators of $E_{0}(H)=\mathbb{C}^{2 m}$. According to [8] we have

$$
W\left(\frac{1}{2} t, U\right)=\operatorname{span}\left(\begin{array}{c}
I_{m} \\
X
\end{array}\right)
$$

where $X \in \mathbb{C}^{m \times m}$ is the unique hermitian solution of (3.1). From Corollary 2.2 we know that $W\left(\frac{1}{2} t, U\right)$ is independent of the choice of $U$. Moreover, $W\left(\frac{1}{2} t, U\right)=W$ where $W$ is given by (3.3). Hence, if $W=\operatorname{span}\left(\begin{array}{c}Y \\ Z\end{array}\right)$ then $Y$ is nonsingular, and

$$
\operatorname{span}\left(\begin{array}{c}
Y \\
Z
\end{array}\right)=\operatorname{span}\left(\begin{array}{c}
I \\
Z Y^{-1}
\end{array}\right)
$$

implies that $X=Z Y^{-1}$ is the solution of (3.1).

\section{Conclusions}

Results of this note can be considered in a module theoretic framework. In a subsequent paper we shall make the connection of Theorem 2.1 with marked subspaces in [4] and with torsion modules over discrete valuation domains in [1].

Acknowledgement. We would like to thank Dr. G. Dirr for a valuable comment.

\section{References}

[1] P. Astuti and H.K. Wimmer, Regular submodules of torsion modules over a discrete valuation domain, to appear in Czechoslov. Math. J.

[2] L. Baratchart, P. Enqvist, A. Gombani, and M. Olivi, Surface acoustic wave filters, unitary extensions and Schur analysis, Report No. 44, 2003, Institut Mittag-Leffler, Stockholm, http://www.mittagleffler.se/preprints/0203s, submitted for publication.

[3] R. Bru, L. Rodman, and H. Schneider, Extensions of Jordan bases for invariant subspaces of a matrix, Linear Algebra Appl. 150(1991), 209226.

[4] J. Ferrer, F. Puerta, and X. Puerta, Geometric characterization and classification of marked subspaces, Linear Algebra Appl. 235(1996), 15-34. 
[5] G. Freiling, On the existence of hermitian solutions of general algebraic Riccati equations, Schriftenreihe d. FB Mathematik der Universität-GHDuisburg, Nr. 446 (1999).

[6] I. Gohberg, P. Lancaster, and L. Rodman, Matrices and Indefinite Scalar Products, Operator Theory: Advances and Applications, Vol. 8, Birkhäuser, Basel, 1983.

[7] I. Gohberg, P. Lancaster, and L. Rodman, Invariant Subspaces of Matrices with Applications, Wiley, New York, 1986.

[8] V. Kučera, Algebraic Riccati equations: Hermitian and definite solutions, in S. Bittanti, A.J. Laub and J.C. Willems (Eds.), The Riccati Equation, pp.53-88, Springer Verlag, Berlin 1991.

[9] P. Lancaster and L. Rodman, Algebraic Riccati Equations, Clarendon Press, Oxford 1995.

[10] A.C.M. Ran and L. Rodman, A class of robustness problems in matrix analysis, in D. Alpay, I. Gohberg and V. Vinnikov (Eds.), Interpolation Theory, Systems Theory and Related Topics, The Harry Dym Anniversary Volume, Operator Theory: Advances and Applications 134, pp. 337 - 389, Birkhäuser, Basel 2002. 